\title{
Regular Neural Network Based Machining Parameters Optimization Model Of NC Machine Tool
}

\author{
WangSanping ${ }^{1}$, ChenJunwen ${ }^{*}$ and YanWei $^{1}$ \\ ${ }^{1}$ school of Machinery and Automation, Wuhan University of Science and Technology, Wuhan, Hubei, 430081, China
}

\begin{abstract}
Energy consumption process is the basis for energy efficiency improvement of machine tools. Most of the existing researches focus on the static modelling of energy consumption of a machine tool; however, there are a few studies that paid attention to that how process parameters influence the energy consumption of machine tools during processing. It is noted that the process parameters can be selected to reduce energy consumption during machining processes without additional investment. In this paper, a characteristic energy consumption model for NC machine tool was proposed. Then, the mapping rule between process parameters and energy consumption of machine tool was studied, and the model was solved with the regular neural network (RNN). Finally, the result was verified with an experiment of milling the surface of aluminium block, which can effectively improve the energy efficiency of machine tool. The experiment results are shown that regular neural network is used to optimize the process parameters and process the same machining characteristics; we analyze the in machining process of machine tool based on the three cutting parameters, and then, a model of energy consumption. We employ to learn, and use this trained model to select optimal parameters.
\end{abstract}

\section{Introduction}

With the widespread use of $\mathrm{NC}$ machine tools, the problem of energy consumption has become a hot research topic nowadays. China's manufacturing industry has been in a state of high energy consumption for a long time, the energy consumption per unit GDP is 2.2 times that of the world average, and 3 4 times that of Japan, Europe, America and other developed countries.[1-2]. Machine tool is the foundation of equipment manufacturing, How to improve the energy efficiency of machine tools is an important trend for the manufacturing industry. NC machine tool plays a major role in the processing and manufacturing industry. NC machine tools play a major role in the processing and manufacturing industry. NC machine tool will consume a large amount of energy in the process of working, and the production of chip, noise and waste cutting fluid will affect the environment. In the past studies, a group of scholars have done different researches in the field of machine tool energy consumption. Kashiwaetal[3] have studied the energy consumption of spindle of NC milling machine. Zhao et al. [4] have studied the energy consumption of machine tools from the process parameters, and put forward that the technical parameters and material removal rate play a positive role in improving the energy efficiency of machine tools. Improved BP neural network were used to optimize cutting parameters [5]. GU W predicted the energy consumption of machine tools using neural network ensemble and Drosophila algorithm. Most scholars have analysed the energy efficiency of machine tools from machine tool processing technology, but few scholars have considered the energy consumption of machine tools combined with machining characteristics. [6]Because the machine tool is in different state during the whole cutting process, according to different state, we can establish machine tool energy consumption model. Due to the different cutting depth, cutting speed and spindle speed, the load of the machine tool is different, the energy consumption of the machine tool is different under different conditions. In common machine tool processing, the influence of different machining features, such as milling surface and drill hole on the energy consumption of machine tools, is difficult to be established because of the nonlinear and too many factors involved. The relationship between different machining characteristics and the energy consumption of $\mathrm{NC}$ machine tool is proposed, and the nonlinear function approximation function of regular neural network is used to establish the mode, training through the experimental data obtained from the cutting. Finally, the objective function is obtained, using the algorithm to find the best processing plan, and provide a good plan for energy consumption control of multi feature parts. Formatting the title, authors and affiliations

The energy consumption of NC milling machine in milling process can be divided into two parts: load independent and load related, Load related energy consumption mainly includes energy consumption of spindle motor and feed motor, In the process of

* Corresponding author's e-mail: WUST_WANG@126.com 
machining the workpiece by NC milling machine, The no-load energy consumption is mainly affected by the setting of machine parameters, and cutting energy consumption is mainly affected by process parameters and workpiece materials, the load independent energy consumption mainly includes the fixed consumption of the machine parts, such as the fan motor, the servo system and the cooling pump, which are only affected by the open / stop state. It can be seen from the analysis that the energy consumption of NC machine tools is composed of the energy consumed by the energy dissipation parts of $\mathrm{NC}$ lathes during the processing of workpiece; it includes energy consumption and basic energy consumption of spindle motor, feed shaft motor and cooling system motor. [7]

In this paper, from the different state of machine tool in the process of actual processing, the energy consumption under the different state of machine tool is divided into four parts in the whole process:

(1) Star-up energy consumption. In the process of actual processing, because the whole system structure of the machine tool is complex, the electrical and mechanical parts are converted from the closed state to the starting state after the machine is opened. This process takes about one minute for the machine to enter the working state. Although the process is short, the experimental results show that the energy consumption of the machine is short. The peak will be reached.

(2) No-load power consumption. In the process of machining, the machine tool is not always contacted with the work piece surface, so the machine tool will be in no load state. In addition to the spindle motor, besides the spindle motor, the $\mathrm{X}$ axis motor, the $\mathrm{Y}$ axis motor and the $\mathrm{Z}$ shaft motor are still in the working state. The main auxiliary appliances are lighting system, chip system, hydraulic system, and cooling system and so on.

(3) Processing energy consumption. During the cutting process, the contact between the tool and the workpiece surface, the greater the cutting force, the greater the power demand, the higher the energy consumption. The energy consumption of the servo motor will be caused by theistic-resistance action of feed shaft and the friction force of the worktable. The energy consumption of the servo motor varies with the change of the feed speed. Besides, the energy consumption of the machining is also related to the cutting speed and the cutting depth. Processing energy consumption accounts for most of the energy consumption in the whole process. This part of energy consumption affects the energy efficiency of machine tools, which is the focus of our attention.

(4) Downtime energy consumption. When the machine tool is finished, the machine tool will change from the running state to the stop state. Although the time of the process is very short, the energy consumption will reach the peak. The four state energy consumption of a machine tool is shown in Figure 1.

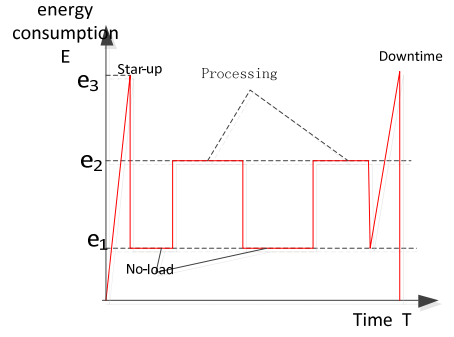

Figure 1. Four state energy consumption of a machine tool

Therefore, the energy consumption model of $\mathrm{NC}$ milling is set up:

$\mathrm{E}_{1}=\int_{0}^{\mathrm{T}_{1}} p_{\mathrm{s}} \mathrm{dt}_{1}(1) \quad \mathrm{E}_{2}=\int_{0}^{\mathrm{T}_{2}} p_{\mathrm{n}} \mathrm{dt}_{2}(2) \quad \mathrm{E}_{3}=$ $\int_{0}^{\mathrm{T}_{3}} p_{\mathrm{p}} \mathrm{dt}_{3}$ (3) $\quad \mathrm{E}_{4}=\int_{0}^{\mathrm{T}_{4}} p_{\mathrm{d}} \mathrm{dt}_{4} \quad$ (4)

$\mathrm{E} 1, \mathrm{E} 2, \mathrm{E} 3$ and E4 respectively indicate the starting energy consumption, no-load energy consumption, processing energy consumption and downtime energy consumption during the whole process. In the actual test, we cannot accurately calculate the proportion of the total energy consumption of each part, in which the two parts of E1 and E4 represent the two processes of machine tool opening and shutdown, and their value is a constant in theory, they are not related to the processing process, so we only need to optimize the total energy consumption. The smaller the value, the smaller the energy consumption and the processing energy, the total energy consumption and the parameters involved can be given by formula (5) :

$$
\left.f_{e}\left(n, v_{f}, a_{p}\right)=\sum_{i}^{n} E i=1,2,3,4\right)
$$

Among them, $n, v_{f}$, and $a_{p}$ denote spindle speed, feed speed and cutting depth respectively. $p_{s} 、 p_{\mathrm{n}} 、 p_{\mathrm{n}}$ 、 and $p_{\mathrm{d}}$ respectively represent the starting instantaneous power of the machine tool, the no-load instantaneous power, the processing instantaneous power, and the downtime instantaneous power, respectively. $t_{1} 、 t_{2} 、 t_{3}$ and $t_{4}$ indicate the time of the instantaneous power of the machine tool in different states, respectively. Because the time of machine starting and stopping is short, most of the machine tools are in the state of empty and processing. Therefore, the energy consumption and processing energy are the main consideration when studying the energy consumption of different processing characteristics.

\section{$2 \quad$ Modelling of energy consumption by regular neural network in processing}

In this section, we will introduce the regular neural network as a powerful tool to mine the hidden relationship between process parameters with energy consumption of NC machine tool. In general, the regular neural network would put several regular items, such as L1 norm and L2 norm, on its weights to avoid complex model during the training process, which is the major difference between the regular neural network with classical neural network. In this paper, the model of regular neural network consists of four layers, including 
one input layer, two hidden layers and one output layer, and its architecture is illustrated at Figure 2.

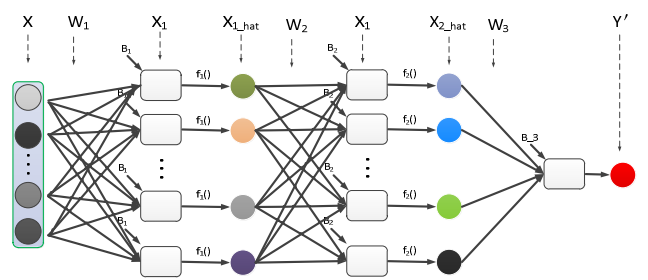

Figure .2 Regular neural network structure diagrams

In the neural network, the spindle speed, feed speed and cutting depth are used as input variables of the neural network, and the total energy consumption of machine tools is the output of neural network.

The first active function on the first hidden layer is sigmoid function, and the second one is linear function. The definitions of these active functions are given as follows:

$$
\begin{aligned}
& f_{1}(x)=\frac{1}{1+e^{-x}} \\
& f_{2}(x)=x
\end{aligned}
$$

and their gradients with regard to $x$ can be computed easily by equations below:

$$
\begin{aligned}
& \frac{\partial f_{1}(x)}{\partial x}=f_{1}(x)\left(1-f_{1}(x)\right) \\
& \frac{\partial f_{2}(x)}{\partial x}=1
\end{aligned}
$$

Given a feature vector $X_{i} \in R^{N_{i}}$, where $N_{i}$ denotes the dimensions of process parameters and is equal to number of neuron on the input layer, and let $y_{i}$ denote energy consumption under the $X_{i}$. Due to that our goal is to learn the mapping rule between process parameters with energy consumption of machine tool, the mean square error (MSE) is selected as loss function of regular neural network, and is given as follows:

$$
l\left(X_{i}\right)=\frac{1}{2}\left(\bar{y}_{i}-y_{i}\right)^{2}
$$

Where $\bar{y}_{i}$ is the predicted value of the model. In addition, we should add related regular items to the objective function of our model. The regular item can be defined as follows:

$$
R\left(W_{1}, W_{2}, W_{3}\right)=\left\|W_{1}\right\|+\left\|W_{2}\right\|+\left\|W_{3}\right\|
$$

Thus, the objective function of the regular neural network can be written as follows:

$$
O\left(X_{i}, W_{1}, W_{2}, W\right)=l\left(X_{i}\right)+\lambda \bullet R\left(W_{1}, W_{2}, W_{3}\right)
$$

Where $\lambda$ is a trade-off between the loss function and the regular item, and $W_{1}, W_{2}$ and $W_{3}$ are weights of the model. In order to optimize these weights, we employ back-propagation algorithm (BP algorithm) as optimization scheme. To this end, we should compute the 'error rate' of each layer, and then, compute gradients with regard to related parameters based on the 'error rate'. Next, we would give a detailed description of process of optimizing parameters by BP algorithm. [8]

First, compute the 'error rate' on the last layer:

$$
\delta_{4}=\mathrm{y}^{\prime}-\mathrm{y}
$$

According to $\delta_{4}$, we can compute gradients with regard to regard to $\mathrm{W}_{3}$ and $\mathrm{B}_{3}$ easily as the following:

$\frac{\partial \mathrm{l}}{\partial \mathrm{W}_{3}}=\delta_{4} \times \mathrm{x}_{2-\text { hat }}^{\prime}+\mathrm{W}_{3}$

$\frac{\partial \mathrm{l}}{\partial \mathrm{B}_{3}}=\delta_{4}$

In same way, the 'error rate' on the third layer can be given as follows:

$$
\delta_{3}=\left(\mathrm{W}_{3} \delta_{4}\right) \circ \mathrm{f}_{2}^{-1}
$$

Where $\circ$ means element-wise multiplication, and gradients with regard to regard to $\mathrm{W}_{2}$ and $\mathrm{B}_{2}$ easily as the following:

$\frac{\partial \mathrm{l}}{\partial \mathrm{W}_{2}}=\delta_{3} \times \mathrm{x}_{1-\text { hat }}^{\prime}+\mathrm{W}_{2} \frac{\partial \mathrm{l}}{\partial \mathrm{B}_{2}}=\delta_{3}$

Final, compute the 'error rate' on the second layer: $\delta_{2}=\left(\mathrm{W}_{2} \delta_{3}\right) \circ \mathrm{f}_{1}^{-1}$

Thanks to computation of $\delta_{2}$, gradients with regard to regard to $W_{1}$ and $B_{1}$ can be given:

$$
\begin{gathered}
\frac{\partial \mathrm{l}}{\partial \mathrm{W}_{1}}=\delta_{2} \times \mathrm{x}_{1}^{\prime}+\mathrm{W}_{1} \\
\frac{\partial \mathrm{l}}{\partial \mathrm{B}_{1}}=\delta_{2}
\end{gathered}
$$

Based on equations above, we can employ Gradient Descent to update the parameters of regular neural network.

\section{Selection of training sample data in experimental scheme}

\subsection{Experimental condition}

Experimental equipment selection NC milling machine XK714D vertical NC milling machine, Selection of common aluminium blocks as experimental materials, the specification of the material is $120 * 80 * 40$. Tool is high-speed steel for cylindrical end milling cutter, the blade diameter is $10 \mathrm{~mm}$, the helix angle is 30 degrees, the front angle is 10 degrees, the rear angle is 15 degrees, and the number of teeth is 4 . The energy consumption monitoring device is a WT1800 high-precision power analyser. As shown in Fig. 3, the power and energy consumption data of the milling machine can be monitored in real time.

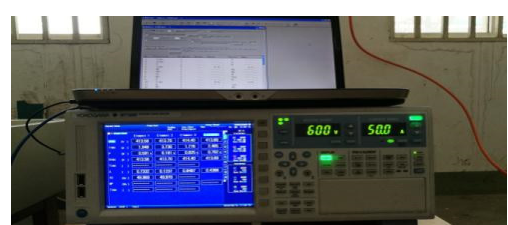

Figure 3 WT1800 high precision power analyser 


\subsection{Experimental scheme}

The theoretical analysis is made, an experimental scheme is proposed and the simulation results are given. The three-dimensional model of the experiment is shown in Figure 4.

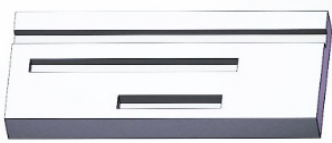

Figure 4 Experimental cutting model

The experimental scheme and part of the energy consumption data are shown in Table 1.

Table 1 Experimental data sheet

\begin{tabular}{ccccc}
\hline $\begin{array}{c}\text { Machining } \\
\text { length } \mathrm{m}\end{array}$ & $\begin{array}{c}\text { Spindle } \\
\text { speed } \mathrm{n} \\
(\mathrm{r} / \mathrm{min})\end{array}$ & $\begin{array}{c}\text { Feed speed } \\
\mathrm{V}_{\mathrm{f}} \\
(\mathrm{mm} / \mathrm{min})\end{array}$ & $\begin{array}{c}\text { Cutting } \\
\text { depth } \mathrm{a}_{\mathrm{p}} \\
(\mathrm{mm})\end{array}$ & $\begin{array}{c}\text { energy } \\
\text { consumption } \\
/ \mathrm{J}\end{array}$ \\
\hline 40 & 600 & 180 & 0.5 & 42669.00 \\
40 & 900 & 270 & 1.0 & 42339.96 \\
40 & 1500 & 450 & 2.0 & 19790.64 \\
40 & 1800 & 540 & 2.5 & 17592.48 \\
80 & 600 & 180 & 0.5 & 81986.76 \\
80 & 1500 & 450 & 2.0 & 41047.2 \\
80 & 1800 & 540 & 2.5 & 38534.4 \\
120 & 600 & 180 & 0.5 & 123465.6 \\
120 & 1200 & 360 & 1.5 & 77018.4 \\
120 & 1500 & 450 & 2.0 & 66344.4 \\
120 & 1800 & 540 & 2.5 & 61459.2 \\
\hline
\end{tabular}

\section{$4 \quad$ Model training and parameter optimization}

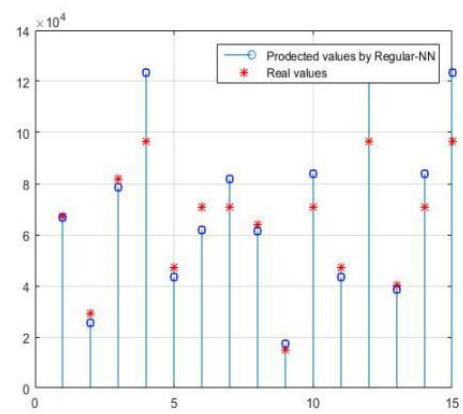

(a)
The model is constructed by the regular neural network method. The model training of the neural network is to train the neural network constructed by the existing data in the case of the model unknown. In the modelling process, the large fraction is trained as a sample, and the model is similar to the actual model with the power of its own adjusting weight. In the process of neural network modelling, another part of data is used to verify the accuracy of the model constructed. All the following simulation results are obtained by Mat lab R2013b running on a laptop with Inter core i7-6500U CPU @ $2.50 \mathrm{GHz}, 8 \mathrm{~GB}$ RAM memory, and 64-bit windows10 OS.

The change cure of loss during iteration is shown in Figure 5. From this picture, we can learn that the regular neural network can quickly achieve convergence at limited steps when we use our data to train the model.

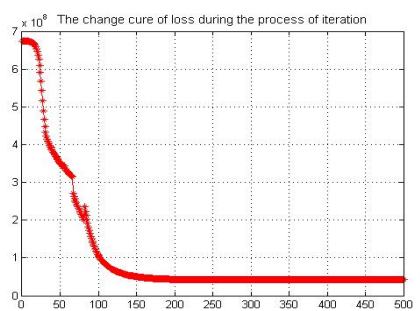

Figure 5 . The change cure of loss during iteration

100 sets of training samples are used to test the model established by the neural network, and compare network output and target output. The results are shown in Figure 6. Through the regular neural network, the optimized cutting parameters are used to predict the energy consumption of the machining features (such as the milling plane). The predicted value and the real value are shown in Figure 7 it can be found that the energy consumption model of NC machine tool established by neural network is feasible and the error is in a very small range.

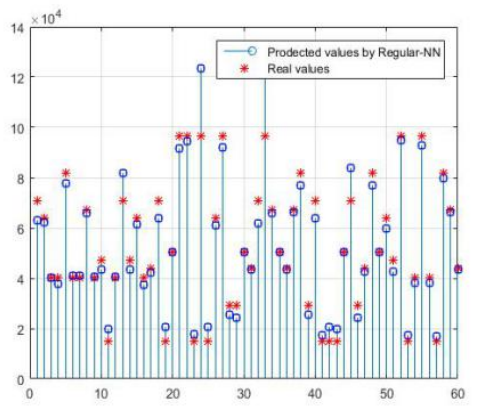

(b)

Figure 6.The comparison between the predicted value obtained by our model and the real value. Fig (a) is on the train dataset, and Fig (b) is on the test dataset.

According to the optimization results, the machining parameters and cutting parameters of the machine tool are: $\mathrm{n}=1200 \mathrm{r} / \mathrm{min}$, feed speed $\mathrm{V}_{\mathrm{f}}=270 \mathrm{~mm} / \mathrm{min}$, cutting depth $a_{p}=1 \mathrm{~mm}$. The prediction value of energy consumption calculated by neural network model is 9.47 $\mathrm{x} 104 \mathrm{~J}$, and the optimized parameters are used to test the milling plane according to the above testing scheme, and the three energy is obtained. The consumption is $9.27 \mathrm{x}$
$10^{4} \mathrm{~J}, 9.18 \times 10^{4} \mathrm{~J}, 9.02 \times 10^{4} \mathrm{~J}$, which is basically consistent with the cutting model.

\section{Conclusion}

The influence of the machining parameters and the cutting parameters on the energy consumption of the machine tool is analysed. A regular network model of the 
energy consumption and processing parameters of the $\mathrm{NC}$ machine tool is established, and the parameters of the target function are optimized. The experimental results are compared with the model prediction results. The results show that the same machining characteristics of the same material can be made to reduce the energy consumption of the machine better when the spindle speed is certain; the low feed speed and the large cutting depth, and the same machining characteristics of the same material. It is only a preliminary study on the energy consumption of machine tools with some processing parameters, and there are many factors affecting the energy consumption of machine tools. The research on the energy consumption of NC machine tools needs to be further studied for the development of "green machine tools".

\section{Acknowledgment}

The research was supported by the Ministry of Science and Technology of the Republic of China under Grant No. 105-1221-E-011-156.

[1] National Natural Science Fund (51775392)

[2] Supported by Hubei Province Key Laboratory of Systems Science in Metallurgical Process (Wuhan University of Science and Technology), (2015X2049),

[3]Centerior Service Science and Engineering, Wuhan University of Science and Technology (CSSE2017GB04)

\section{References}

1. WANG Q , L, HUANG W D, CHEN Z. Online monitoring and analysis system for energy efficiency of NC machine tools [J]. Modern Manufacturing Engineering, 2015, 3(1):39-47.

2. DAI X D, WANG X, BI X G, Interpretation of world energy supply and demand in 2015[J]. Natural gas and petroleum, 2017, 35(1):1-4.

3. Kashiwa H, Sato R, Hayashi A, et al. S131011 Energy Consumption of Spindle and Feed Drive Systems of NC Machine Tool in End-milling Operation[J]. Journal of Cleaner Production, 2013, 2013:_S131011-1-_S131011-5.

4. ZHAO G, WANG Q, RUAN D. Analysis of power efficiency for dry milling machine based on response surface of machining parameters [J]. China Mechanical Engineering, 2016, 27(21):2944-2948.

5. Jayasinghe J, Nadishan K. Neural network based state of charge (SOC) estimation of electric vehicle batteries [J] University of Moratuwa, 2014, 4 (12): 1-4.

6. CAI Wei, LIU Fei, et al An energy management approach for the mechanical manufacture industry through developing a multi-object energy benchmark $[\mathrm{J}]$.Energy Conversion and Management,2017,132:361-371.
7. Kant G, Sangwan K S. Predictive Modelling for Energy Consumption in Machining Using Artificial Neural Network [ J ] ProcardiaCrip, 2015, 37: 205-210.

8. VINCENT AizebeojeBlowguns, PAULTarisaiMativenga.Modelling of Direct Energy Requirement in Mechanical Machining Processes [J].Journal of Cleaner Production, 2013,41:179-186. 